\title{
Smart Phone Application on MIMO (Organ Donation)
}

\author{
Pramoda Devi B, Priya M O, Varsha S Sannur, Vinutha C K, Raviraj P* \\ Department of Computer Science and Engineering, GSSS Institute of Engineering and \\ Technology for Women, Mysuru, India
}

DOI: https://doi.org/10.21467/proceedings.1.8

* Corresponding author email: raviraj@gsss.edu.in

\begin{abstract}
The number of people who die due to the delays incurred in obtaining an organ or lack there of is high. Without a more accessible procedure to obtain the necessary organ, additional delays are incurred, and when dealing with such a sensitive domain, any reduction with regards to the time can potentially save a life. The primary objective of the Organ Donation app is to bring about a direct means of communication between both a patient and a donor, thereby ignoring the necessity of a middleman and creating a more accessible approach to both the patient and the donor. Through clear logging of potential donors by location and presenting these options to the patient at the time of need, the process of obtaining an organ for a transplant can go about in a smooth manner, while removing the possibility of any delays that could potentially be introduced in the normal process.
\end{abstract}

Keywords: Mobile Application, organ donor, organ recipient, healthcare, NGO, organ donation;

\section{INTRODUCTION}

With the growing interest in the quality of life, healthcare has become one of the fields that have received increasing attention. Especially with the increase of population, the burdens of healthcare are continuously increasing. This means that the need for communication between family, friends and community members to share in healthcare activities. On top of this, there is a very strict constraint in time. Donors are usually persons who have been involved in serious accidents and are kept artificially alive though cerebral death has been stated. In such patients is extremely difficult to maintain acceptable vital parameters for a long time, and any variation in them can lead to the loss of organs.

Moreover, after the organs have been taken from the body, they can be stored for very few hours and the transplant must take place in the shortest period possible. Currently, the activities described above are almost entirely performed by human beings, with weak supports to process large amounts of data and to coordinate with each other. The organization and storage of information in compact, re-usable ways and the introduction of software support

(C) 2018 Copyright held by the author(s). Published by AIJR Publisher in Proceedings of the $3^{\text {rd }}$ National Conference on Image Processing, Computing, Communication, Networking and Data Analytics (NCICCNDA 2018), April 28, 2018.

This is an open access article under Creative Commons Attribution-NonCommercial 4.0 International (CC BY-NC 4.0) license, which permits any non-commercial use, distribution, adaptation, and reproduction in any medium, as long as the original work is properly cited. ISBN: $978-81-936820-0-5$ 
systems in the process of matching can accelerate and simplify the assignment of organs. In the last decade, mobile, computer, and communications technologies have been evolving rapidly. Using mobile for search or access to information through internet network becomes one of a necessary part of every daily life. There are many networks that are connected to each other and many mobile application used by people to implement various types of work. In addition, smartphones contain many services which include an internal database, voice recognition, GPS and ability to connect to local wireless networks and the Internet that can be used to develop applications that can be used in healthcare

Organ supply process at a specific time is an important factor in medicine and treatment and often considered as a very important element in determining life or death of the patient. Therefore, the need emerged here to provide an effective way to overcome this problem The technological development happened in the process of making smart phones in terms of their size and diversity of services offered and their widespread application among people and provide Internet service even in remote locations with the ability to locate people through these cell phones in order to find a solution to the problem of providing blood for patients who need them within a short time which helped greatly in saving the lives of many patients The process of donating organ can only occur through people that are alive and that the patient's life depends on quickly and easily finding and locating organ donor. This study works on creating an integrated and easy to use system in determining the where about of organ donors through mobile phones.

The aim of the application is to design and implement a organ donor mobile application which can provide the ability to search for the organ donor in application database and also give the ability to search for the donor through the internet to locate the nearest donor centers in case the organ seeker cannot find a donor in the database of application. In addition, the application can identify the nearest hospital and also determine the presence or absence of a certain doctor at the hospital as you can specify the competence of doctors currently in the hospital. When the application is launched at the first time, the Home Screen Activity is started, which has all the tabs and menu item to access different activities.

\section{LITERATURE SURVEY}

[1] P Priya,2014 Introduced an extended web application to timely update the information regarding the donors, acceptor and patients where the administrator accesses the whole information about blood bank management system. Also, the work has a Push technology with security, to protect the contact details of the donors in a web application where it can be misused by third parties. It also maintains the amount of each available blood group, if the stock of a particular blood group is lower than the required amount then the proposed method notifies the donor to donate blood. In addition to the web application, an android mobile application is proposed to search the donors who are available nearby during the emergency

Proceedings of the $3^{\text {rd }}$ National Conference on Image Processing, Computing, Communication, Networking and Data Analytics (NCICCNDA 2018) 
Smart Phone Application on MIMO (Organ Donation)

cases such as accidents. [2] R. Vanitha, 2013., presented BCloud Blood Donor Contact Manager Web Services that allows you to maintain a free, easy-to- organize database of contacts and their blood groups. With this convenient-and potentially lifesaving--app, you can quickly check your Android device for someone with a certain blood type and contact them immediately.

[3] Sultan Turhan,2013 presented a smart phone's application for the volunteer blood donor to increase the willingness and accessibility with the purpose of providing a continuous blood supply. This application helps healthcare centers to provide the blood as quick as possible when their stocks are insufficient. The application sends periodically.

[4] Lisa Maria Garnweidner-Holme, 2015 Introduced designing and developing a smartphone application (the Pregnant+ app) that automatically transfers blood sugar levels from the glucometer and has information about healthy eating and physical activity. This formative research included expert group discussions among health professionals, researchers, and experts in data privacy and security. User-involvement studies were conducted to discuss prototypes of the app. Results indicated that the content of the application should be easy to understand given the varying degree of patients' literacy and in line with the information they receive at clinics. The final version of the app incorporated behavior change techniques such as self monitoring and cues to action. Results from the first round of interactions show the importance of involving expert groups and patients when developing a mobile health-care device.

[5] M. V. M. Figueredo, 2004 described the implementation of a telemedicine system for patient monitoring using mobile telephony. The major aspect of this application is its generality, which allows the use of any patient monitor with a RS-232 interface. The system proved to be quick and reliable. Therefore, it represents an applicable solution to tele-home care.

[6] Rajapraveen .K.N,2012, presented a topic called "Health Care Application in mobile device" mobile itself a plenty of applications as a repository, on adding the new health care application in mobile, it provides the user knowledge of disaster management of health problems.

[7] Javed Akhtar Khan, 2015 introduced the new working concept of a blood bank management system for the rural area. Information and computer technology are very famous in blood banks for its potentials in working efficiency as well as service quality we just provide new facilities for all blood bank management as well as for seeker. It plays a vital role in this new concept. the main objective behind in this concept is more of places blood bank does not have a good facilities for storing a blood unit in bank for large time period, but with the help of this new concept blood bank have to provide a blood to any time and any situation to seeker apart from that seeker is also able a call the donor for blood in a very serious condition of patient. 


\section{PROPOSED METHOD}

We have two sides for this project: One is the Android application itself, and the second is the server that responds to requests. So, on the Android app, aka Mimo, We have a login screen, and a registration screen. If the user selects the Registration option, he/she is sent to another "Activity" which is another screen. Here, we ask for the user details like First/Last name, email, password (which will be encrypted using MD5 hash) The hospital they're residing in, ID proof, Gender, and Blood Type. The last two are important for organ compatibility.

On the login screen, once the user submits the information, a connection is made to our Server (you will need the Internet for this), and we rely on our server to do the authentication for us. The result is returned back to the app, and the app then decides which Activity to load based on the role of the user (donor or patient).

If the user was a donor, the donor activity is loaded and information is passed to that screen (information about the donor like blood type and gender, for example). The donor then selects the organ he/she wishes to donate. After this is done, upon submit, we set up a connection with the server to store these details, after which the Donor is successfully added to the waiting list.

On the Patient's end, the patient is presented with a drop-down box of organs. Upon selecting the organ he/she needs, a customized or filtered list of the available organs are presented to the patient. Again, this information is first sent to the server, and the server, in turn, returns all the details necessary to the patient's instance of the app. This step is complex in execution. After the patient confirms the organ/donor details, information is sent back to the server for storage. This information is passed to the hospital who can set up an operation date by contacting the donor directly.

As you can see, the Android app and the Server needs to work together to get Mimo to be functional. We are not using a local DB (like SQLite or Volley) because the nature of the app demands that the data should be distributed online.

\subsection{Algorithm for MIMO (Organ donation):}

Step 1: Start

Step 2: The user has to install the application.

Step 3: Select the user type(Donor/Patient).

Step 4: The user has to fill necessary credentials (name Contact info,gender,blood group,id proof).

Step 5:Notify which organ he wishes to donate/receive.

Step 6: The patient has to notify when the organ is needed.

Step 7: Admin will locate the nearest donor and notify the patient.

Proceedings of the $3^{\text {rd }}$ National Conference on Image Processing, Computing, Communication, Networking and Data Analytics (NCICCNDA 2018) 
Smart Phone Application on MIMO (Organ Donation)

Step 8: The doctor/NGO will contact the donor directly

And fix the operation date.

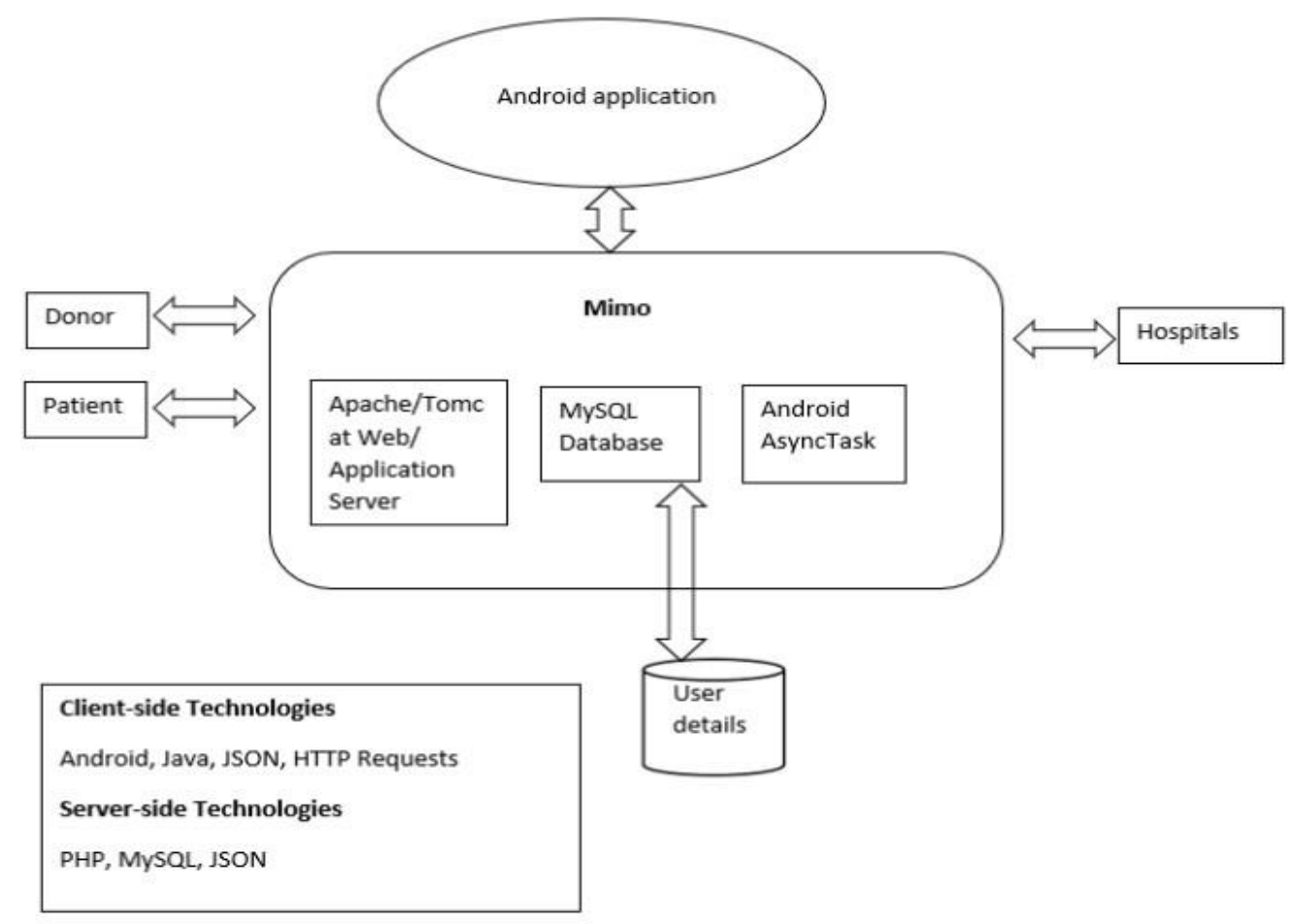

Figure 1: Architectural diagram

\section{CONCLUSION}

The proposed system contributes significantly to saving lives through easily and quickly locating the persons donating human organs and locate the nearest hospital and doctor. All the goals can be achieved by building a vast network of people who want to donate organ as you can use this network to increase people's awareness about the importance of donating organs to save lives. The proposed system provides an easy and flexible interface so that all persons at various levels can use the proposed system through the use of smart phones with Internet and GPS property. Proposed system can further be customized according to different needs of different health systems. This can be customized for the needs of organ donation authorities and rules and regulations in that specific area. That will increase the usability and effectiveness of this application. 


\section{REFERENCES}

[1] P. Priya , V. Saranya , S. Shabana and Kavitha Subramani.(2014). The Optimization of Blood Donor Information and Management System by Technopedia, International Journal of Innovative Research in Science, Engineering and Technology, 390-395

[2] R.Vanitha and P.Divyarani. (2013). BCloud App: Blood Donor Application for Android Mobile, International Journal of Innovations in Engineering and Technology (IJIET), 2, 396-401.

[3] Sultan Turhan. (2013). AN ANDROID APPLICATION FOR VOLUNTEER BLOOD DONORS, Computer Science \& Information Technology (CS \& IT), 23-30.

[4] Lisa Maria Garnweidner-Holme , Iren Borgen, Iñaki Garitano , Josef Noll and Mirjam Lukasse.(2015). Designing and Developing a Mobile Smartphone Application for Women with Gestational Diabetes Mellitus Followed-Up at Diabetes Outpatient Clinics in Norway,Healthcare journal, 310-323.

[5] Figueredo MV1, Dias J.(2004). Mobile telemedicine system for home care and patient monitoring,Conf Proc IEEE Eng Med Biol Soc

[6] Rajapraveen K.N and Dr. N.K.Prasanna Kumari.(2012). Health Care Application in Mobile, 2, 11, 2012

[7] Javed Akhtar Khan and M.R. Alony.(2015). A New Concept of Blood Bank Management System using Cloud Computing for Rural Area (INDIA)", International Journal of Electrical, Electronics and Computer Engineering,20-26.

Proceedings of the $3^{\text {rd }}$ National Conference on Image Processing, Computing, Communication, Networking and Data Analytics (NCICCNDA 2018) 